\title{
Successful Treatment of Epstein-Barr Virus-Induced Necrotizing Retinitis with Intravitreous Ganciclovir, Foscarnet, and Methotrexate
}

\author{
Junsang Cho ${ }^{a} \quad$ Abdhish R. Bhavsar ${ }^{b}$ Daniel H. Cho ${ }^{c}$ Victoria Sattarova ${ }^{d}$ \\ Anne S. Abel ${ }^{\mathrm{e}}$ Rebecca Zadroga ${ }^{f}$ \\ aUniversity of Missouri-Columbia School of Medicine, Columbia, MO, USA; ${ }^{b}$ Retina Center \\ of Minnesota, Minneapolis, MN, USA; 'Dartmouth College, Hanover, NH, USA; dUniversity of \\ Minnesota, Minneapolis, MN, USA; 'Department of Ophthalmology, Hennepin Healthcare, \\ Minneapolis, MN, USA; fDepartment of Infectious Disease, Hennepin Healthcare, \\ Minneapolis, MN, USA
}

Keywords

Necrotizing retinitis - Epstein-Barr virus - Methotrexate

\section{Abstract}

This is a report of an immunocompromised 49-year-old renal transplant patient with EpsteinBarr virus (EBV)-induced necrotizing retinitis (NR). The patient with NR underwent diagnostic vitrectomy. Polymerase chain reaction (PCR) testing of the vitreous fluid was positive for EBV $(25,000 \mathrm{IU} / \mathrm{mL})$ and negative for all other organisms. The patient was treated with intravitreous ganciclovir and foscarnet. After only mild clinical improvement in retinitis and an increased quantitative EBV PCR $(69,000 \mathrm{IU} / \mathrm{mL})$, intravitreous methotrexate was added to the aforementioned intravitreous antiviral injections. After eight rounds of ganciclovir/foscarnet and three injections of methotrexate, the NR resolved, the quantitative EBV PCR decreased to $29 \mathrm{IU} / \mathrm{mL}$, and the patient's visual acuity improved. To our knowledge, this is only the second case report to demonstrate efficacy of intravitreous methotrexate in an immunocompromised patient with EBV-induced NR. Intravitreous methotrexate combined with ganciclovir and foscarnet may be an effective treatment strategy for patients with PCR-positive EBV-induced NR that does not respond to conventional antiviral therapy. 


\section{Introduction}

Epstein-Barr virus (EBV), also referred to as human herpes virus-4, is a herpes virus with lifetime prevalence of acquisition that is over 95\% [1]. Infection is transmitted by aerosol or direct nasopharyngeal contact. While asymptomatic in children, it can commonly cause fever, fatigue, sore throat, adenopathy, and hepatosplenomegaly in adults. In addition, EBV can be associated with lymphoid or epithelial malignancies including nasopharyngeal carcinoma, gastric carcinoma, and B-cell malignancies (Burkitt, Hodgkin, and vitreoretinal lymphoma) [1].

Unfortunately, there is a paucity of literature exploring the ocular manifestations of EBV. A few studies have examined intraocular EBV-DNA levels and EBV antibodies in patients with uveitis [2-4]. Only a handful of EBV-induced necrotizing retinitis (NR) cases in the setting of EBV infection have been reported (Table 1) [5-9]. Furthermore, the treatment of EBV-associated NR has not been fully elucidated.

Advances in molecular analytics, including genomic screening and polymerase chain reaction (PCR), allow clinicians to identify the specific viral strain in NR cases with accuracy and specificity. $\mathrm{NR}$ is most commonly caused by varicella-zoster virus (VZV) and herpes simplex virus (HSV) types 1 and 2 [11]. Although most NR cases lack definitive demonstration of EBV as the sole etiologic virus, there have been a handful of reports that identify EBV as the sole cause by qualitative PCR [5-9].

In this report, we describe a case of isolated EBV-induced NR diagnosed by quantitative PCR in the setting of immunosuppression. The clinical presentation, diagnosis, and management are described to increase awareness and treatment options for EBV-induced NR.

\section{Case Presentation}

A 49-year-old female with a history of P-anti-neutrophil cytoplasmic antibody (P-ANCA) positivity and a renal transplant secondary to membranous nephropathy presented with a blind spot in her left eye that was present for 3 weeks. She denied any eye pain, flashes, or floaters

Table 1. Summary of PCR-confirmed Epstein-Barr virus-induced acute retinal necrosis

\begin{tabular}{|c|c|c|c|c|}
\hline Author & Age/gender & Immunocompromised? & Treatment & Outcome \\
\hline $\begin{array}{l}\text { Hershberger et al. } \\
\text { (2003) [10] }\end{array}$ & $10 \mathrm{mo} / \mathrm{M}$ & $\begin{array}{l}\text { Yes, X-linked } \\
\text { lymphoproliferative } \\
\text { disorder }\end{array}$ & $\begin{array}{l}\text { Bone marrow } \\
\text { transplant }\end{array}$ & Resolution \\
\hline Hamam et al. [9] & $46 \mathrm{yr} / \mathrm{M}$ & $\begin{array}{l}\text { Yes, high-dose systemic } \\
\text { corticosteroids, } \\
\text { methotrexate, cyclosporine, } \\
\text { and intravitreal steroids for } \\
\text { chronic uveitis }\end{array}$ & $\begin{array}{l}\text { PO valacyclovir/ } \\
\text { hydrochloride and } \\
\text { trimethoprim/ } \\
\text { sulfamethoxazole }\end{array}$ & Resolution \\
\hline Schaal et al. [7] & $55 \mathrm{yr} / \mathrm{F}$ & $\begin{array}{l}\text { Yes, oral methylprednisolone } \\
\text { and infliximab for rheumatoid } \\
\text { arthritis }\end{array}$ & $\begin{array}{l}\text { PO valacyclovir/ } \\
\text { hydrochloride, topical } \\
\text { corticosteroids }\end{array}$ & Enucleation \\
\hline Roquelaure et al. [8] & $72 \mathrm{yr} / \mathrm{M}$ & No & $\begin{array}{l}\text { PO acyclovir, } \\
\text { IV ganciclovir }\end{array}$ & $\begin{array}{l}\text { Unknown, } \\
\text { patient } \\
\text { deceased }\end{array}$ \\
\hline Mashima et al. [5] & $83 \mathrm{yr} / \mathrm{F}$ & $\begin{array}{l}\text { Yes - oral methylprednisolone } \\
\text { for interstitial pneumonia and } \\
\text { chronic pyelonephritis }\end{array}$ & $\begin{array}{l}\text { IV ganciclovir, IV } \\
\text { acyclovir, intravitreous } \\
\text { methotrexate }\end{array}$ & Resolution \\
\hline
\end{tabular}

M, male; F, female; PO, oral; IV, intravenous; mo, month; yr, years. 
in the left eye. Her immunosuppressive medications included $360 \mathrm{mg}$ of mycophenolate mofetil twice daily and $5 \mathrm{mg}$ oral prednisone daily. Additional pertinent medical history included hypertension treated with hydralazine and carvedilol and thyroid disease treated with levothyroxine. She also had a history of idiopathic intracranial hypertension, well-controlled with topiramate.

Visual acuity in the right eye was $20 / 20$ and $7 \mathrm{ft} / 200$ in the left eye. She had a left relative afferent pupillary defect. Slit-lamp examination of the right eye was unremarkable. Slit-lamp examination of the left eye was significant for mild subconjunctival hemorrhage, rare anterior chamber cell, and 2+ vitreous cells.

Dilated fundus exam of the right eye was physiologic. Dilated fundus exam of the left eye revealed vitreous strands and snowballs inferiorly, $1+$ disc edema, and a large area of retinitis and retinal hemorrhage with periphlebitis inferiorly consistent with necrotizing retinitis. The left macula was diffusely edematous (Fig. 1a). Macular optical coherence tomography (OCT) of the left eye showed cystoid macular edema and subretinal fluid with a central macular thickness of 842 microns (Fig. 2a, b). Fluorescein angiography showed perivenous leakage and late diffuse hyperfluorescence in the macula and in the areas of NR. Macular OCT and fluorescein angiography of the right eye were unremarkable.

The initial differential diagnoses included VZV, HSV, CMV, Toxoplasma gondii, EBV, syphilis, or tuberculosis-induced NR. Extensive blood work was performed. Infectious labs included QuantiFERON TB Gold, FTA-ABS, Bartonella henselae and quintata IgM and IgG, Borrelia burgdorferi, T. gondii IgM and IgG, Toxocara IgG, and HIV antibody titers, which were all negative or nonreactive. Autoimmune workup included anti-nuclear antibodies, rheumatoid factor, angiotensin-converting enzyme, lysozyme, erythrocyte-sedimentation rate, C-reactive protein, C-anti-neutrophil cytoplasmic antibodies (C-ANCA), P-ANCA, myeloperoxidase antibody, proteinase 3 antibody IgG, HLA-A29, and HLA-B27 and was significant for elevated C-reactive protein $(0.84 \mathrm{mg} / \mathrm{dL})$, erythrocyte-sedimentation rate $(65 \mathrm{~mm} / \mathrm{h})$, myeloperoxidase antibodies (53.1 CU), P-ANCA (1:640), and lysozyme $(13.8 \mu \mathrm{g} / \mathrm{mL})$.

Four days after initial presentation, the patient underwent 23-gauge pars-plana vitrectomy and an undiluted vitreous biopsy sample was tested for cytology, silver stain, PCR for VZV, HSV1 and HSV2, CMV, T. gondii, TB, and EBV. Intraoperatively, the patient received intravitreous injections of ganciclovir $2.0 \mathrm{mg}$, foscarnet $2.4 \mathrm{mg}$, clindamycin $1 \mathrm{mg}$, penicillin $300 \mathrm{IU}$, and dexamethasone with partial air-fluid exchange. Cytology of the vitreous fluid showed sparse mixed inflammation and was negative for malignancy. A lymphoproliferative process was ruled with the negative vitreous cytology. PCR testing of the vitreous fluid was positive for EBV (25,000 IU/mL) and negative for CMV, HSV1, HSV2, and VZV. A concurrent PCR of the serum was ordered which showed a viral load of $<390$ copy $/ \mathrm{mL}$ and quantitative $\log <2.6$ which was considered a negative result.

The patient was diagnosed with EBV-induced NR and treated with weekly intravitreous injections of ganciclovir $2.0 \mathrm{mg}$ and foscarnet $2.4 \mathrm{mg}$ starting the week following the vitrectomy. Prednisolone acetate was started at the patient's follow-up visit after her first ganciclovir/ foscarnet injection. After two injections, the NR had only slightly improved, and her visual acuity was $9 \mathrm{ft} / 200$ in the left eye. After consultation with the infectious disease and renal transplant teams, mycophenolate mofetil was reduced to $180 \mathrm{mg}$ twice daily. Repeat vitreous biopsy with quantitative PCR showed an increased EBV titer of 69,000 IU $/ \mathrm{mL}$. Although there was no monoclonal B- or T-cell population in the vitreous to suggest EBV-associated lymphoma, the patient was treated with intravitreous methotrexate $400 \mu \mathrm{g}$ since it is believed to inhibit EBV-DNA replication through interference with EBV-DNA polymerase [5].

The patient was then treated with alternating weekly intravitreous injections of ganciclovir $2.0 \mathrm{mg} /$ foscarnet $2.4 \mathrm{mg}$ and methotrexate $400 \mu \mathrm{g}$ in addition to renally dose-adjusted oral ganciclovir $450 \mathrm{mg} \mathrm{q} 48 \mathrm{~h}$ after her first ganciclovir/foscarnet injection and continued

\section{Karger'}




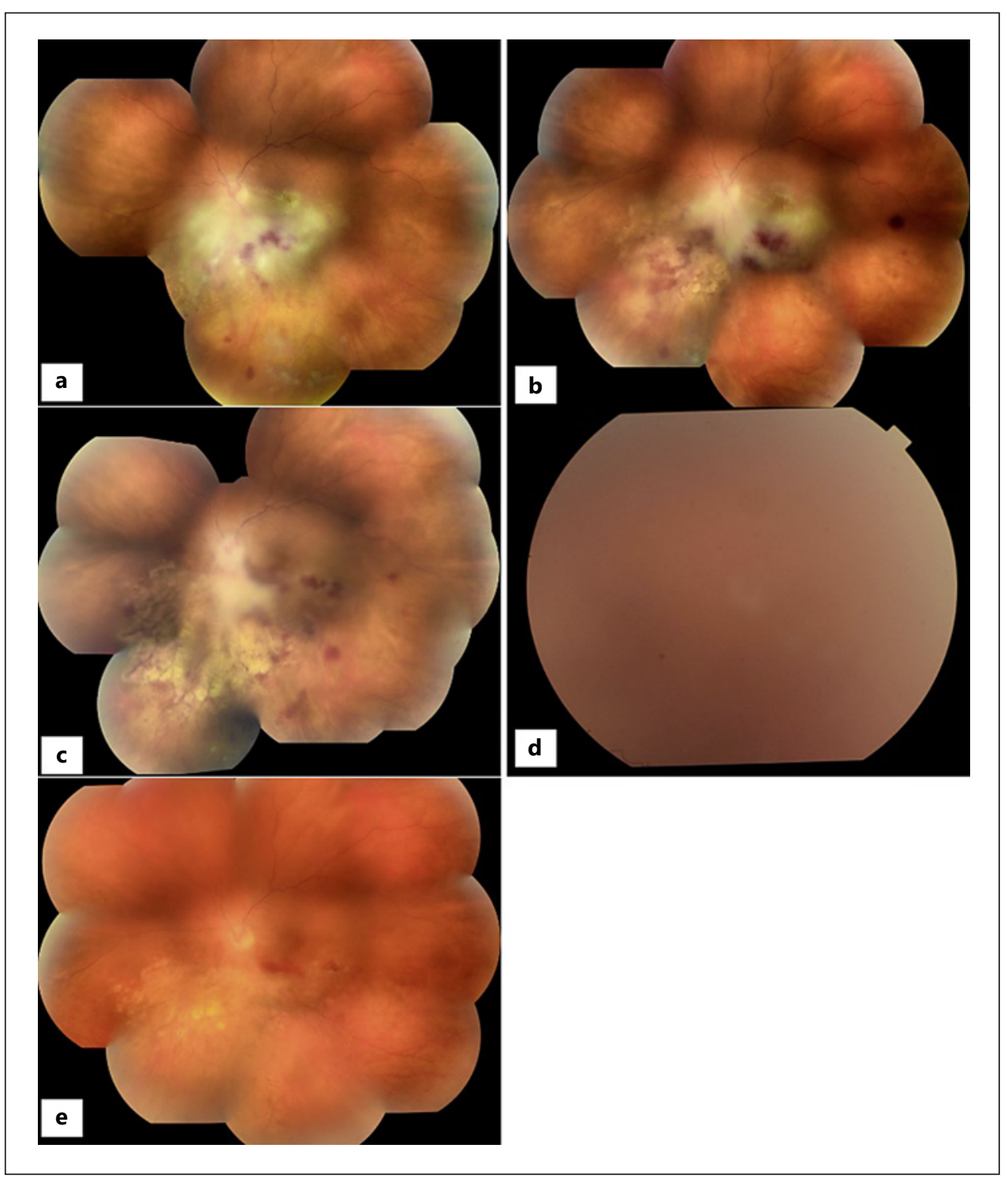

Fig. 1. Serial fundus photographs showing changes in fundus appearance and improvement of NR over time in the left eye. a Active retinitis, periphlebitis, and retinal hemorrhage at presentation. $\mathbf{b}$ Slight improvement of retinitis at inferonasal border of active retinitis after two rounds of intravitreous ganciclovir/foscarnet injections. c Moderate improvement in retinitis 1 week after second intravitreous methotrexate injection and three intravitreous ganciclovir/foscarnet injections. d Vitreous hemorrhage after three injections of ganciclovir/ foscarnet and methotrexate. e Regression of retinitis and periphlebitis after 7 intravitreous ganciclovir/foscarnet injections and 3 intravitreous methotrexate injections.

until her seventh ganciclovir/foscarnet injection. Her visual acuity initially improved to $20 / 400$ after the first ganciclovir/foscarnet injection. After 3 cycles of the aforementioned alternating treatment, vitreous quantitative PCR was 9,700 IU/mL. However, the patient developed a hyphema and vitreous hemorrhage as well as corneal epitheliopathy, and visual acuity decreased from $20 / 400$ to hand motion in the left eye. Prednisolone acetate $1 \%$ drops were continued at a frequency of four times daily, and methotrexate was discontinued. Corneal epitheliopathy resolved after a few weeks, and there was no evidence of retinal breaks as is seen in cases of necrotizing retinitis caused by other herpes viruses. 


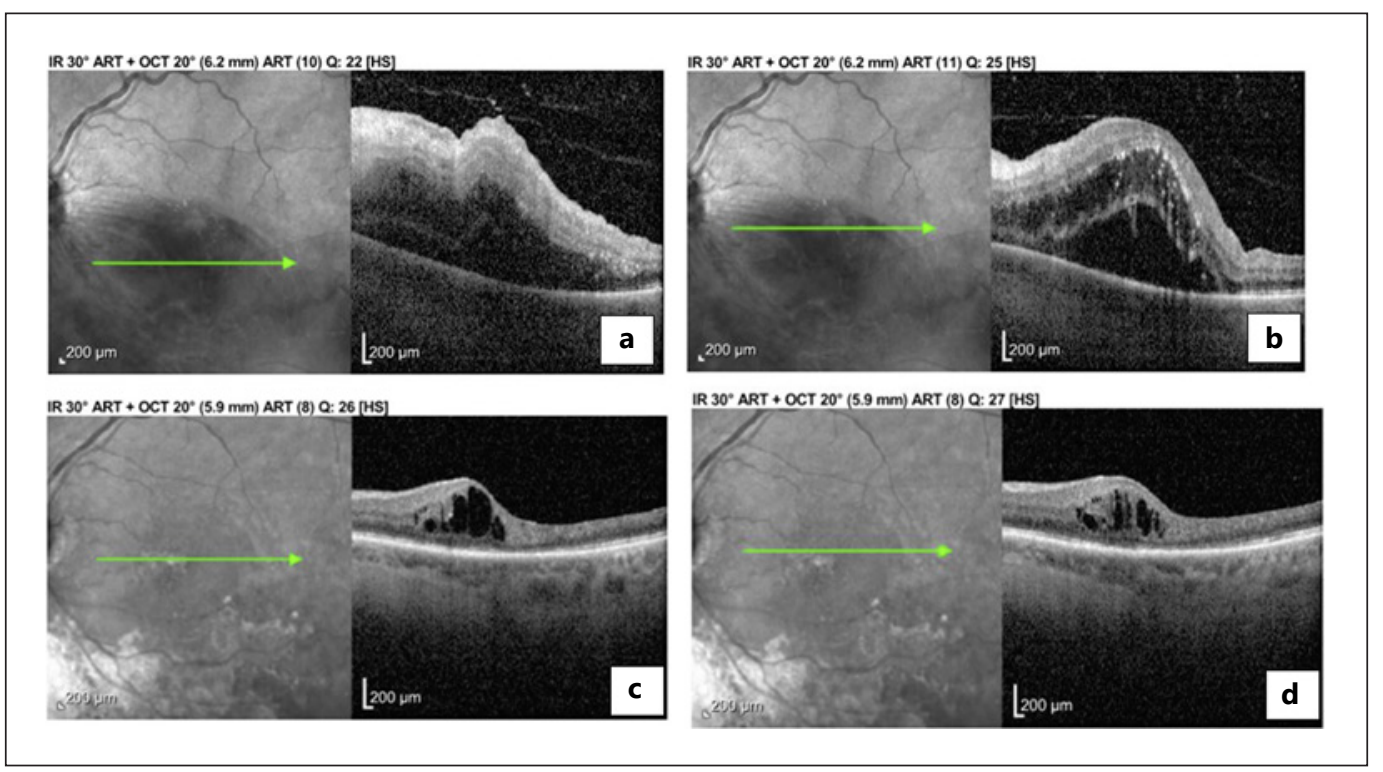

Fig. 2. OCT images at presentation with NR showing macular thickening, outer retinal disruption, subretinal fluid, and edema (a, b) and at most recent follow-up visit showing resolution of retinal thickening, subretinal fluid, outer retinal disruption, and improving macular edema, as well as new temporal retinal thinning (c, d).

At the time of the fifth intravitreous injection of ganciclovir and foscarnet, 2 weeks after the third methotrexate injection, quantitative EBV PCR showed a markedly reduced viral load (29 IU/mL). Four weeks later, her left eye visual acuity improved to $20 / 100$, improving to 20/80 with pinhole. Fundus exam showed regression of the white-yellow areas of NR (Fig. 1a-e). OCT showed improvement in retinal architecture, resolution of subretinal fluid, and improvement in macular edema (Fig. 2a-d). To date, the patient has received eight injections of ganciclovir/ foscarnet and three injections of methotrexate. One additional intravitreous ganciclovir/ foscarnet injection is planned in 1 month.

\section{Discussion}

The clinical features of intraocular EBV infection are not fully elucidated because most cases are asymptomatic [1]. In addition, the detection of HSV1, EBV, and human herpes virus 6 via vitreous biopsy is not always associated with clinical disease. Furthermore, EBV infection has not been readily reported in the literature to cause uveitis, vitritis, papillitis, or vasculitis. As a result, the pathogenicity of EBV in NR cases has been debated.

EBV is a ubiquitous virus affecting the majority of the population [11]. The most common clinical manifestation is mononucleosis, but EBV can also cause lymphoproliferative disorders like Burkitt's lymphoma. The major host cells of EBV are B lymphocytes, and infected B cells are persistent throughout life. While the EBV in infected B cells is mostly latent, immunosuppression may trigger reactivation of EBV which results in replication of the virus and evasion of immune system surveillance.

Treatment of EBV-induced NR has traditionally involved various oral and intravenous antiviral drugs. In general, oral and intravenous acyclovir is effective in treating initial and recurrent HSV and VZV infections through inhibition of the herpes-specified DNA polymerase, which inhibits HHV proliferation yet does not eliminate the cells already infected. Antiviral therapy has not been widely accepted for systemic EBV treatment because most symptoms and 
signs of infectious mononucleosis are not due to the virus itself, but rather from the body's immune response. Systemic EBV symptoms are more attributable to immunopathic responses to EBV-infected cells, particularly EBV-infected B lymphocytes that circulate in the blood and infiltrate tissues of affected organs. Consequently, steroid therapy has been used to treat EBV retinitis, but only with varying degrees of success [11].

In addition to antiretroviral therapy and steroids, clinicians have also attempted early prophylactic laser retinopexy and/or early vitrectomy for nonspecific NR. There has also been an EBV-induced NR case reported that did not respond to therapy and eventually resulted in enucleation [11]. In the absence of clear EBV treatment protocols and the resistant nature of EBV, early sampling of the aqueous and/or vitreous with PCR should be considered in all patients with suspected EBV retinitis.

Our patient initially received intravitreal ganciclovir and foscarnet. However, since the RN improved only slightly, her treatment regimen was modified to include alternating methotrexate and ganciclovir/foscarnet injections as well as systemic ganciclovir and reduction in mycophenolate. Although the intravitreal methotrexate injections were discontinued after the development of corneal epitheliopathy, the resolution of her retinitis improved once methotrexate was added to the intravitreal antiviral treatment. The reduced viral load cannot be solely attributable to methotrexate, but this case demonstrates utility in augmenting traditional intravitreous antiviral therapy with methotrexate for the treatment of EBV-induced NR.

There have been very few studies analyzing the effectiveness of methotrexate in the management of intraocular EBV. As a result, there is a paucity of the literature exploring the treatment regimen for patients who fail antiretroviral therapy due to the rarity of the condition. Mashima et al. [5] reported the first case of successful EBV-induced NR treatment with intravitreous methotrexate. Similar to our patient, Mashima et al. [5] initially treated EBV-induced NR with antiviral therapy including both intravenous acyclovir and ganciclovir. With an increase in viral load and worsening clinical necrosis, the patient completed fifteen intravitreous methotrexate injections - same dose as our patient $(400 \mu \mathrm{g})$ - leading to resolution and no evidence of side effects including corneal epitheliopathy.

To our knowledge, this is the third case report of PCR-proven NR due to EBV in an immunocompromised patient and the second report of EBV-induced NR effectively treated with methotrexate. We describe a case of EBV-associated NR diagnosed via PCR from the vitreous fluid without detection of DNA from other herpes viruses. Furthermore, our patient's serum EBV viral load was within normal limits, which provides convincing evidence that replication of EBV took place within the eye. Similar to Mashima et al. [5], successful treatment was noted by serial decreases in PCR titers over time following the addition of methotrexate, which has been shown to correlate with clinical outcomes [11]. While the mechanism of response to intravitreous methotrexate has not been elucidated, it may serve as potential treatment in resistant cases of EBV-associated NR through inhibition of EBV-DNA replication via interference with EBV-DNA polymerase [5]. It is extremely unlikely that this case was due to a lymphoproliferative process since vitreous cytology showed no evidence of malignancy, and only three intravitreous injections of methotrexate were administered which typically would not be enough to result in elimination of the necrotizing retinitis. Furthermore, quantitative PCR testing showed reduced vitreous EBV titers as the necrosis resolved.

We acknowledge that our patient received intravitreous ganciclovir, foscarnet, and oral ganciclovir in addition to the intravitreous methotrexate injections. She also had reduction in immunosuppression through reduction of mycophenolate dose. Consequently, the exact therapeutic contribution of each agent is unknown. However, the patient's clinical picture improved only slightly with traditional intravitreal antiviral therapy and improved substantially after intravitreous methotrexate was added to the treatment regimen. We believe this report provides evidence for the addition of intravitreous methotrexate in treatment-resistant EBV-induced NR.

\section{Karger'}


Further studies are needed to determine which patients are most likely to benefit from the addition of intravitreous methotrexate to the traditional antiviral treatment including ganciclovir and foscarnet for EBV-induced NR. We hope this case report assists clinicians in the treatment of EBV-induced NR that is refractory to antiviral treatment.

\section{Statement of Ethics}

Written informed consent was obtained from the patient for publication of this case report and any accompanying images. The current study adhered to the tenets of the Declaration of Helsinki. Ethics approval was not required for this study in accordance with local/national guidelines.

\section{Conflict of Interest Statement}

The authors declare that there are no conflicts of interest to disclose.

\section{Funding Sources}

This research received no specificgrant from any funding agency in the public, commercial, or not-for-profit sectors.

\section{Author Contributions}

J.C., A.R.B., D.C., V.S., A.S.A., and R.Z.: writing and reviewing.

\section{Data Availability Statement}

All data generated or analyzed during this study are included herein. Further inquiries can be directed to the corresponding author.

\section{References}

1 Cohen JI. Epstein-Barr virus infection. N Engl J Med. 2000 Aug 17;343(7):481-92.

2 Keorochana N, Treesit I, Funarunart P. Characteristics and clinical outcomes of hypertensive anterior uveitis. Ocul Immunol Inflamm. 2020 May 18;28(4):538.

3 Chen Y, Xu H. Epstein-Barr virus-associated hemophagocytic lymphohistiocytosis following cyclosporine for uveitis. Ocul Immunol Inflamm. 2020 May 18;28(4):549.

4 Imai A, Takase H, Imadome K-I, Matsuda G, Ohnishi I, Yamamoto K, et al. Development of extranodal NK/T-cell lymphoma nasal type in cerebrum following Epstein-Barr virus-positive uveitis. Intern Med. 2017;56(11):1409-14.

5 Mashima A, Usui Y, Umazume K, Muramatsu D, Goto H. Successful treatment of necrotizing retinitis with Epstein-Barr virus-positive ocular fluid by intravitreal methotrexate injection. Ocul Immunol Inflamm. 2020 May 18;28(4):552.

6 Grossniklaus HE, Aaberg TM, Purnell EW, Luka J, Seemayer TA. Retinal necrosis in X-linked lymphoproliferative disease. Ophthalmology. 1994 Apr;101(4):705.

7 Schaal S, Kagan A, Wang Y, Chan CC, Kaplan HJ. Acute retinal necrosis associated with Epstein-Barr virus: immunohistopathologic confirmation. JAMA Ophthalmol. 2014 Jul 1;132(7):881.

8 Roquelaure D, Harbarth S, Schutz JS, Thumann G, Chronopoulos A. Epstein-Barr-virus-assoziierte akute retinale nekrose. Ophthalmologe. 2016 Oct 23;113(10). 
9 Hamam RN, Mansour A, El Mollayess G. Positive Epstein-Barr virus polymerase chain reaction in a case of acute retinal necrosis. Can J Ophthalmol. 2012 Dec;47(6):e61-2.

10 Hershberger VS, Hutchins RK, Witte DP, Schneider S, Harris RE, McGonegle SJ. Epstein-Barr virus-related bilateral acute retinal necrosis in a patient with X-linked lymphoproliferative disorder. Arch Ophthalmol. 2003;121(7):1047-9.

11 Lau CH, Missotten T, Salzmann J, Lightman SL. Acute retinal necrosis features, management, and outcomes. Ophthalmology. 2007 Apr;114(4):756. 